\title{
Procesos de reestructuración y verticalización en el centro de Concepción: Barrio Condell
}

Restructuring and verticalization processes in Concepción's downtown: Barrio Condell

\section{Leonel Pérez Bustamante}

Departamento de Urbanismo, Universidad de Concepción, Concepción, Chile.

\section{Gerardo González Bello}

Becario CEDEUS, Facultad de Arquitectura, Urbanismo y Geografía, Universidad de Concepción, Concepción, Chile.

\section{Daniela Villouta Gutiérrez}

Asistente de investigación CEDEUS, Facultad de Arquitectura, Urbanismo y Geografía, Universidad de Concepción, Concepción, Chile. villoutadaniela@gmail.com

\section{León Pagola Contreras}

Asistente investigación CEDEUS, Facultad de Arquitectura, Urbanismo y Geografía, Universidad de Concepción, Concepción, Chile.

\section{Christian Ávila Zañartu}

Asistente investigación CEDEUS, Facultad de Arquitectura, Urbanismo y Geografía, Universidad de Concepción, Concepción, Chile.

\section{Resumen}

El edificio en altura implica el retorno a un tipo arquitectónico bajo nuevas formas de construcción, de usos de suelo y de tipo de ocupación, constituyéndose como unidades de análisis urbano que afectan el tejido urbano y a los actores presentes. La investigación buscó comprender las transformaciones morfológicas originadas por procesos de verticalización y los efectos en las formas de habitar de residentes del centro urbano de Concepción. Por ello, a partir del estudio de caso del barrio Condell, se analiza el uso de suelo residencial en altura mediante levantamientos planimétricos $y$ entrevistas semiestructuradas a residentes según el año de llegada al barrio. Los resultados muestran que la aparición del edificio en altura en el tejido urbano implica un tipo de ocupación y uso del suelo que altera sustantivamente el paisaje urbano y algunas formas de habitar, situando la movilidad cotidiana como aspecto clave. El trabajo permite abrir una línea de trabajo para estudios especializados que aporten a la reorientación de la planificación y los Instrumentos de Planificación Territorial (IPT) en ciudades intermedias.

Palabras clave: ciudad neoliberal, formas de habitar, reestructuración urbana, verticalización.

\begin{abstract}
The building in height implies a new form of construction, land use and occupation type, constituting itself as urban analysis units that affect the urban fabric and the actors present in it. This research seeks to understand the morphological transformations caused by verticalization processes and the effects on the ways of inhabiting residents of the urban center of Concepción. Therefore, based on the case study of the Condell neighborhood, the use of residential land in height is analyzed by planimetric surveys and semi-structured interviews of residents according to the year of arrival in the neighborhood. The results show that the appearance of the building in height in the urban fabric implies a type of occupation and land use that substantially alters the urban landscape and the ways of living, placing everyday mobility as a key aspect. The research allows to open veins for specialized studies that contribute to the reorientation of planning and Territorial Planning Instruments (IPT) in intermediate cities.
\end{abstract}

Keywords: neoliberal city, urban renewal, verticalization, ways of inhabiting.

Documento recibido el 24 de julio de 2019 y aceptado el 16 de diciembre de 2019.

Resultados del trabajo realizado en el Centro de Desarrollo Urbano Sustentable (CEDEUS - FONDAP №15110020).

Cómo citar: Pérez, L., González, G., Villouta, D., Pagola, L. y Ávila, C. (2019). Procesos de reestructuración y verticalización en el centro de Concepción: Barrio Condell. Revista de Urbanismo, 41, 1-17. https://doi.org/10.5354/0717-5051.2019.53926 


\section{Introducción}

Los procesos de reestructuración urbana de áreas centrales constituyen transformaciones de los espacios urbanos y el entorno construido a nivel social, económico y político. En muchos casos se representa principalmente mediante el concepto de gentrificación, en donde los medios de comunicación generan una visión sesgada frente a este fenómeno y lo caracterizan únicamente como un proceso de sofisticación urbana (López-Morales, 2013), consecuencia de la llegada de nuevos integrantes con poder adquisitivo y riqueza cultural, desplazando a una población existente de menores recursos económicos (Inzulza y Galleguillos, 2014). Clark (2005) nos permite comprender mas globalmente el fenómeno como la reestructuración espacial de un área urbana mediante la inyección de capital fijo en mercado inmobiliario y de infraestructura, orientada al reemplazo de usuarios de ingresos medios-bajos por usuario de poder económico superior, en un contexto de mercantilización del suelo.

El Barrio Condell es un espacio claramente definido dentro de la ciudad de Concepción caracterizado por tener una relevancia histórica, que se inscribe en una de las zonas de Renovación Urbana declaradas en el Plan Regulador vigente desde 2004, ocupando 15 manzanas compuestas por 2.662 habitantes según el Censo 2017. El barrio aparece en la trama urbana como un sector rodeado por avenidas estructurantes, lo que hace que sea imperceptible el contexto que guarda dentro de sus calles y antigua plaza con más de cien años (inaugurada oficialmente en 1905). Este lugar, con construcciones de fachada continua, y algunas calles de adoquines que aún resisten las mejoras realizadas los últimos años, dan cuenta del abandono que ha sufrido el barrio en las últimas décadas. Ya en los años $80^{\prime}$ del siglo pasado se hablaba dentro de la prensa local respecto al estado de abandono que inspiraba la plaza del lugar y la falta de vida que existía. Este hecho, se vio acompañado por la construcción a un costado del barrio Remodelación Paicaví, basada en principios de arquitectura y urbanismo modernos, que le quitaron relevancia al Barrio Condell y su característica plaza.

En general, la Plaza Condell, espacio primado de este sector, es una pieza urbana cargada de valor patrimonial para la comunidad penquista y que ha sido reconocido por parte de la Municipalidad de Concepción, a través del programa Quiero Mi Barrio (2011). Mediante este programa se elaboró un documento de trabajo que resaltó la importancia del barrio y acciones concretas para mejorar el sector y afianzar las percepciones barriales. A pesar de estas acciones, esta pieza urbana no está exenta del crecimiento de la ciudad y está contenida dentro del cuadrante de Renovación Urbana de Concepción. La aparición de proyectos residenciales en altura, dentro del sector y en las cercanías, ha iniciado un debate respecto al impacto de la altura de las masas construidas en la escala del barrio y en las dinámicas barriales y urbanas (Pérez, 2017).

Los efectos de la reestructuración urbana en altura sobre el suelo central y pericentral de grandes metrópolis han sido estudiados exhaustivamente, pero poco se han abordado en ciudades de tamaño medio, ni desde la política pública, ni desde un enfoque socio espacial.

Se plantea la hipótesis de que el proceso de reestructuración urbana del área central de Concepción se puede observar a partir del desarrollo inmobiliario generado en los últimos años, teniendo como consecuencia más visible la proliferación de torres de habitación tipo departamentos de gran altura las que no han considerado los impactos en las formas de habitar de residentes antiguos del sector. A su vez, la inversión en infraestructura de transporte, la liberalización de la normativa y los subsidios a la compra de vivienda en áreas centrales han sido relevantes para impulsar estas transformaciones urbanas en barrios que han sido históricamente conformados por viviendas de baja altura. La lógica economicista en el discurso y práctica pública, o urbanismo proempresarial, constituye los marcos jurídicos y económicos que, por un lado, aseguran y aumentan el potencial de ganancia de agentes inmobiliarios (López-Morales, Gasic y Meza, 2012, 2014) y por otro alteran formas de habitar de residentes antiguos.

Así, resulta necesario conocer el impacto de la edificación en altura que ha proliferado en los últimos años en los barrios centrales y en torno a ejes viales estructurantes, para posibilitar que se tomen en cuenta estas externalidades y así construir instrumentos y una planificación territorial que considere estos efectos. Bajo esta premisa surgen interrogantes ¿Cómo y de qué manera han afectado las transformaciones morfológicas que han ocurrido en el Barrio Condell a las formas de 
habitar de residentes antiguos del barrio? ¿Cuál es la percepción de los habitantes del barrio y de los edificios frente a la presencia de estos edificios en altura insertos en el Barrio Condell?

Se reconoce que la ciudad de Concepción no ha quedado exenta de este fenómeno urbano a nivel nacional y se encuentra experimentando un proceso de densificación en altura en barrios centrales y pericentrales de baja altura. Por lo anterior, el objetivo principal de esta investigación radica en analizar las transformaciones morfológicas originadas por los procesos de reestructuración urbana a partir de la aparición del edificio en altura en el Barrio Condell, y cómo estas transformaciones generan efectos socio espaciales en las formas de habitar de residentes antiguos del barrio.

\section{Marco teórico}

La neoliberalización de las ciudades constituye un proceso que no obedece a un patrón lineal, es dinámico y se ha proyectado a través de las épocas, en conjunto con un sistema económico y político validado en distintos sectores de la sociedad. Además, responde a ensamblajes políticos particulares derivados de contextos sociohistóricos específicos asumidos en la implantación del neoliberalismo a escala urbana. Para el caso chileno, resulta clave evidenciar que es el país en donde se inician las políticas de shock neoliberal (Klein y García, 2007), expresadas a nivel urbano en la liberalización que la dictadura en 1979 del mercado del suelo (Sabatini, 2000). Así, se puede plantear que las políticas urbanas en las ciudades chilenas han vivido más de tres décadas de influjo y hegemonía neoliberal aplicado sin contrapesos, situando una tendencia específica de transformación social y política, que afecta a las ciudades latinoamericanas en las últimas dos décadas, consistente en la proliferación de políticas urbanas que tienen como objetivo (explícito o implícito) desplazar y expulsar población de las áreas centrales (Sequera \& Janoschka, 2015). Estas expulsiones estarían definiendo, de modo estructural, la conformación del mundo actual pues más allá del empobrecimiento y las persistentes dinámicas de la desigualdad social, estaríamos en presencia de lógicas expulsivas en variadas escalas locales y regionales, algunas menos visibles que otras, por ejemplo: la expulsión de proyectos de vida y medios de vida, la destrucción de barrios completos y/o su transformación con grandes edificios de departamentos, la expulsión de trabajadores de bajos salarios de los programas gubernamentales de salud y seguridad social, pero también el estigma de grupos vulnerables y precarizados en barrios de la periferia urbana.

En los años noventa, la regulación estatal chilena comienza a tener relevancia y toma un rol facilitador en la introducción del capital inmobiliario, en las áreas centrales de las ciudades más importantes del país, que comenzaron a ser destino del capital en base a un urbanismo empresarial (Harvey, 1989). En este contexto, los gobiernos locales, mediante ajustes normativos de uso de suelo, buscan atraer proyectos inmobiliarios, apoyados por una lógica subsidiaria propuesta por el estado y aplicada a través del Subsidio de Renovación Urbana que data de 1991, estimulando el mercado de suelo y subsidiando el acceso a una propiedad en zonas céntricas (Pérez, Hidalgo, Añasco y Alvarado, 2019). Para autores como Michael Janoschka (2011), el neoliberalismo y la globalización son dos caras de una reestructuración económica que ha introducido enormes cambios en las condiciones generales de la política urbana y en los modos de percibir, diseñar, vivir y gestionar las ciudades contemporáneas, dando paso a una reestructuración urbana. Asimismo, esto ha desembocado en la forma que adoptaron visualmente los espacios y quienes buscan habitarlos en un sentido estrictamente de fin comercial, pensando en su plusvalía generada a partir de la estética que se logra entre los elementos que conforman dicho paisaje urbano y los lugares donde se busca construir (De Mattos, 2004).

Ortiz, Castro y Escolano (2002) plantean que las transformaciones morfológicas y funcionales asociadas a la reestructuración económica y urbana muestran una ruptura con el espacio urbano preexistente, revalorizando la importancia estratégica de la infraestructura para la movilidad y la localización. En este contexto, las nuevas necesidades de movilidad suelen saturar los sistemas existentes, por lo que es necesaria su modernización y reordenación. Es aquí donde la accesibilidad del suelo cobra valor y urgencia. Sin embargo, los procesos de reestructuración urbana de áreas centrales y sus efectos han sido insuficientemente estudiados en ciudades chilenas aparte de la capital nacional y que tienen el carácter de ser capitales regionales o metrópolis con más 
ciudades constitutivas, como es el caso del Barrio Condell en Concepción, sur de Chile.

En las últimas tres décadas al interior de los estudios urbanos, se va configurando la mirada de un urbanismo crítico que da cuenta del impacto de las políticas neoliberales en la forma de construir y habitar ciudad, poniendo énfasis en la situación de desregulación de la planificación y captura de plusvalía a nivel urbano por parte del sector privado. Esto recientemente ha sido conceptualizado como ciudad neoliberal (Hidalgo \& Janoschka, 2014) o urbanismo neoliberal, donde el modelo de restructuración urbana se adapta y subordina a las demandas de una economía global. En ese marco, el Estado asume un rol de promotor de las condiciones legales, políticas y económicas que tienden a favorecer la plena realización de proyectos urbanos que promueven el valor de cambio por sobre la equidad urbana y el valor de uso y apropiación del espacio (Lefebvre, 1968). Brenner y Theodore (2002) muestran las principales características del modelo de desarrollo urbano neoliberal: (i) creación de redes de negocios dirigidas por colaboraciones públicoprivadas; (ii) nuevas formas de política económica local que fomentan la cooperación entre las empresas; (iii) implementación de programas basados en la comunidad para paliar la exclusión social; (iv) promoción de nuevas formas de coordinación interinstitucional y creación de redes entre las distintas esferas anteriores a la intervención estatal local; y (v) formación de nuevas instituciones regionales que promueven la coordinación intergubernamental y el marketing urbano, las que, en convergencia con otros factores, dan lugar a una reestructuración económica y urbana.

La verticalización de la ciudad es un fenómeno estudiado por autores (Fedele y Martínez, 2015; Inzulza y Galleguillos, 2014; Vergara-Vidal, 2017) que en su mayoría convergen al definirlo como la aparición de edificios en altura, en su mayoría residenciales, dentro de los núcleos centrales y pericentrales de las principales ciudades intermedias de Latinoamérica y Chile, densificando el área de forma explosiva. En el caso de Argentina, la verticalización es uno de los cambios más visibles que ha sufrido el tejido urbano de los núcleos centrales de sus ciudades intermedias en las últimas décadas. Ante esto, la aparición del edificio en altura en la planta urbana implicó una nueva modalidad constructiva y un tipo de ocupación que alteró el paisaje urbano y las formas de habitar
(Fedele y Martínez, 2015). Vergara (2017) establece que las primeras edificaciones en altura fueron en su mayoría costeadas y utilizadas por Bancos, Cajas y aseguradoras, y muy pocas de ellas fueron destinadas a fines residenciales. El destino de los edificios en altura estaba más relacionado a funciones administrativas y comerciales, posibilitadas por las nuevas tecnologías, como el ascensor y el desarrollo de la industria del acero y el vidrio (Fedele y Martínez, 2015). Sin embargo, los datos analizados evidencian que este aspecto se ha invertido $y$, en los últimos 25 años, la mayor cantidad de superficie edificada en altura, por sobre los nueve pisos, corresponde a uso residencial, siendo esta la forma elegida para densificar las comunas centrales de la ciudad (Vergara Vidal, 2017).

La edificación en altura pone en marcha desarrollos de densificación y/o compactación de tejidos urbanos. La verticalización de áreas centrales es parte de una transición hacia formas más densas, con la consecuente redefinición de la situación urbana preexistente (Silva, 2004; Costa, 2000). Las operaciones inmobiliarias tienen un peso tal en el desarrollo urbano, que ya tienen modelos tipológicos que han sido probados innumerables veces y que resultan rentables como negocio. Estos ocupan la parcela al máximo, para sacar el mayor aprovechamiento del suelo y que eso se traduzca en mayor cantidad de departamentos para la venta y así lograr la recuperación del capital invertido, en el menor tiempo posible. Este mercado inmobiliario hace que muchas veces la distinción entre un edificio y otro se de sobre la base de su localización dentro de la trama, lo que lleva a que los departamentos se hayan convertido en un producto de características industriales, desde el proyecto hasta la construcción (Fedele y Martínez, 2015).

En las últimas dos décadas las áreas metropolitanas chilenas han experimentado una acelerada verticalización y densificación residencial, lo que genera variados conflictos urbanos. La verticalización, es un fenómeno reciente, pero no nuevo en el mundo. Se presentan aspectos bastante distinguibles en el rápido incremento de escala de construcción, densidad y aglomeración de edificios, así como se perciben efectos ambientales negativos y desvalorización de barrios (Colegio de Arquitectos de Chile, 2018).

El Colegio de Arquitectos de Chile (2018) declara que las casas, y especialmente los departamentos, cada vez 
tienen un costo más elevado, son menos espaciosos, y en edificios cada vez más saturados (en alta densidad) y con mayor impacto en los barrios que los rodean.

Los edificios en altura resultan unidades desagregadas, dividas y dispersas en el tejido urbano. Muchas de las construcciones, que generalmente superan los diez niveles de altura, son construidas en parcelas entre medianeras, de frentes reducidos, lo que implica una fuerte presencia visual como grandes muros ciegos, que no poseen una búsqueda desde la forma, lenguaje o materialidad (Fedele y Martínez, 2015). Una definición aplicable a nuestro territorio y acorde a lo que a simple vista se puede observar frente a los edificios y proyectos inmobiliarios en altura en nuestro país, muchas de las construcciones carecen de expresión y/o intención arquitectónica reduciéndolo a tipologías estándares, repetidas y anónimas. El nicho inmobiliario, son hogares unifamiliares, matrimonios jóvenes con un hijo o simplemente rentistas, que ven en el emplazamiento del edificio ventajas de conectividad respecto de la articulación trabajo-centralidad-residencia. En este sentido López et al. (2014), indican que las inmobiliarias generan un producto homogéneo, sobre el cual se ofertan unidades de acotadas dimensiones, inclusive llegando a producir un noventa por ciento tipologías de departamentos de uno y dos dormitorios, excluyendo en gran medida a grupos familiares más extensos.

El edificio en altura está distante de ser un elemento puntual alabado como signo de progreso e indicador de avance, como lo fue en un comienzo. Actualmente "se ha vuelto un elemento generalizable en el tejido con un significado negativo adherido al tradicional positivo vinculado con el desarrollo económico y de signo modernizador. Este desplazamiento ha reposicionado el debate y actualizado los paradigmas que guían la discusión urbana" (Fedele y Martínez, 2015, p. 85).

Se hace necesario definir a los edificios en altura como unidades que tienen su propio funcionamiento y son actores principales dentro de un fenómeno asociado a la escala urbana y masiva del habitar en una altura construida (Allen, 2013; Gehl, 2010, 2011). Considerar a los edificios de altura como unidades de análisis urbano no sólo implica considerar el efecto de su forma sobre la escala urbana (Brenner, 2004; Cuthbert, 2006), sino también el efecto que el tejido de actores (Ingold, 2007, 2013) implicados en su funcionamiento tiene sobre la escala urbana y sobre su entorno (como se cita en Vergara Vidal, 2017).

\section{Caso de estudio: Barrio Condell, Concepción}

La verticalización, la densificación y la proliferación del edificio residencial en altura configuran un fenómeno que se evidencia a simple vista en la ciudad de Concepción, pero que recientemente comienza a generar debate dentro de las comunidades barriales $y$, más recientemente, académica.

El centro y las peri-centralidades de la ciudad de Concepción no han estado exentas de esta situación, y en ellas es posible evidenciar procesos simultáneos entre el modelo de urbanismo empresarial como el definido por David Harvey (1989), que ha acrecentado la productividad inmobiliaria en altura partiendo de políticas y estrategias de renovación urbana (López, 2008). Esto, a partir de grandes intervenciones urbanas destinadas al reciclaje de espacios centrales en deterioro, que buscan aprovechar las ventajas de localización y, de esta forma, potenciar el mercado inmobiliario y la recirculación del capital.

El Censo del 2017 mostró resultados que evidencian un importante desarrollo de proyectos inmobiliarios en altura durante los últimos quince años (Figura 1). En la Figura 2 , se puede evidenciar que existe un cambio en la modalidad residencial. Sectores antes ocupados por familias de estratos medios-altos, hoy se presentan como enormes edificios que densifican la población, esto se complementa con lo informado por Eduardo Torres, geógrafo de DemoData, quien sentencia: "Concepción tiene un centro dinámico, propio de una ciudad en construcción" (Cisterna, 2018). 


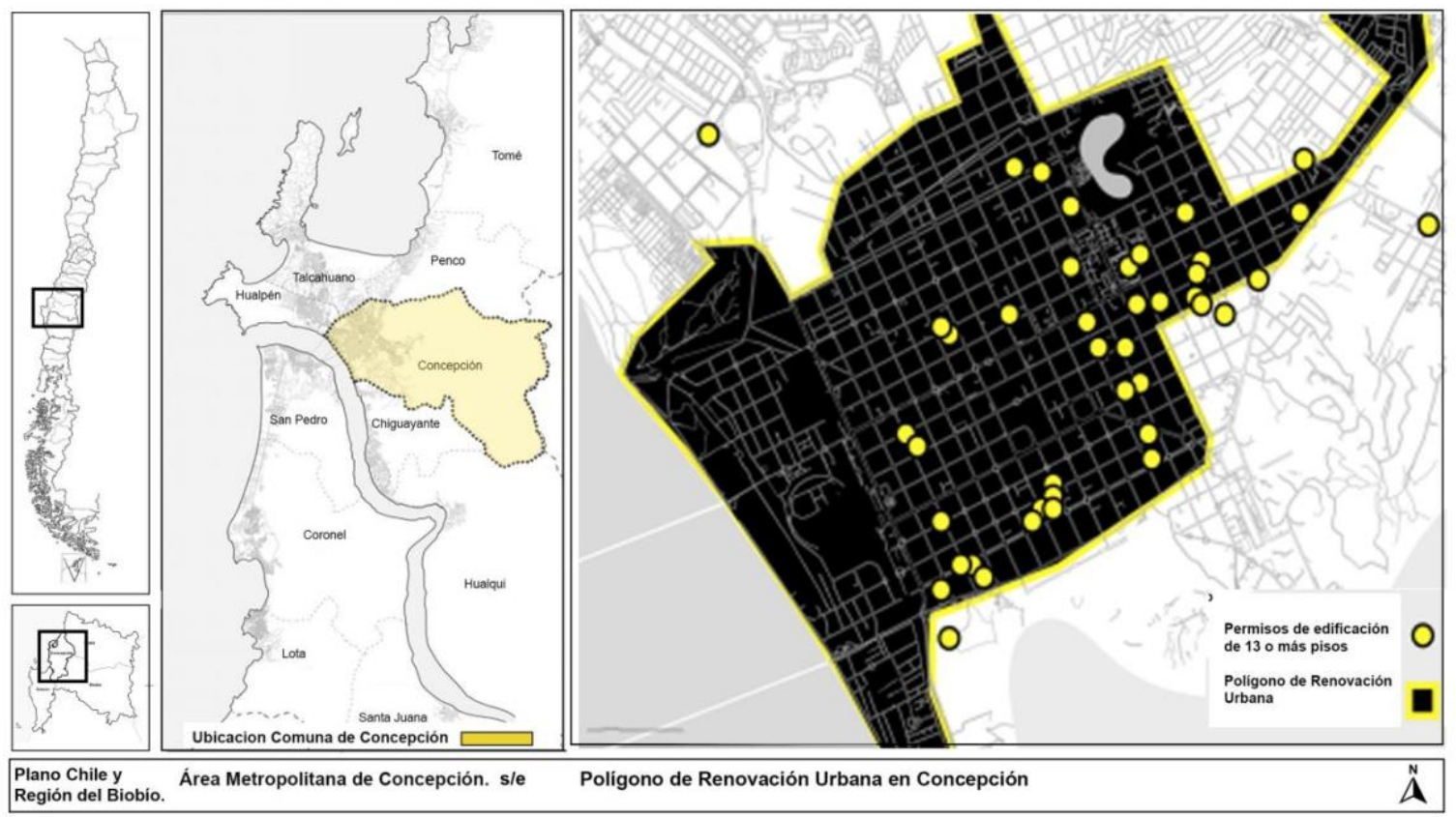

Figura 1. Ámbito geográfico de estudio y localización de permisos de edificación para edificios de 13 pisos o más, 2010 a 2018 en el Polígono de Renovación Urbana en Concepció. Fuente: Elaboración propia a partir de INE 2018.

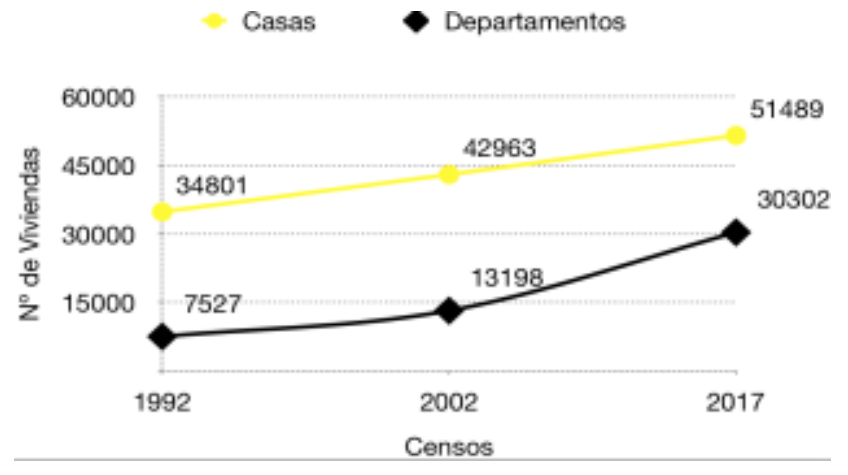

Figura 2. Evolución del número de viviendas particulares en el área urbana de la comuna de Concepción a través de los diferentes censos, a partir de la información entregada por el Instituto Nacional de Estadísticas. Fuente: Elaboración propia.

Fedele y Martínez (2015) declaran que, para las ciudades centrales de Argentina, y asimilable al caso chileno, las operaciones inmobiliarias se encuentran concentradas en el núcleo central, pero sin una planificación previa, más bien las edificaciones en altura se hallan dispersas sin una continuidad espacial y conforman finalmente sectores urbanos heterogéneos. La resultante es la dispersión de los edificios en altura dentro de un área urbana a partir de la intermitencia de la renovación parcela a parcela. Esto produce la ruptura de tejidos urbanos, su alteración principalmente de altura y el dificultoso encastre de nuevas tipologías surgidas en el proceso de densificación. En la misma investigación plantean que estos efectos que están haciendo visible la construcción en altura son los que llevan hoy a cambiar la percepción frente al proceso de densificación.

Es así que el indicador simbólico de proceso de la forma urbana deja de condensarse en la única presencia del edificio en altura, para desplazarse a otros aspectos que justamente lo rechazan: el respeto por el patrimonio, la preservación de características morfológicas del tejido existente y los usos del espacio urbano. (Fedele y Martínez, 2015, p. 69)

También la prensa local está evidenciando que la regulación de las alturas de las edificaciones ha comenzado a producir debate y genera preocupación dentro de las comunidades barriales y a llamar la atención de autoridades comunales. Según artículos publicados por Radio Bío Bío y Diario de Concepción (Cisterna, 2018), como prensa local, la ciudad de Concepción se encuentra en un proceso de modificación de las normas urbanísticas, limitando la altura de los edificios en zonas cercanas al 
núcleo central de la ciudad, para así mitigar los efectos de la construcción en altura. Además, en vista de la aparición de proyectos inmobiliarios de gran envergadura en manzanas que antes no se encontraban densificadas, genera y abre un debate amplio y con opiniones divididas frente al tema (Figura 3 ).
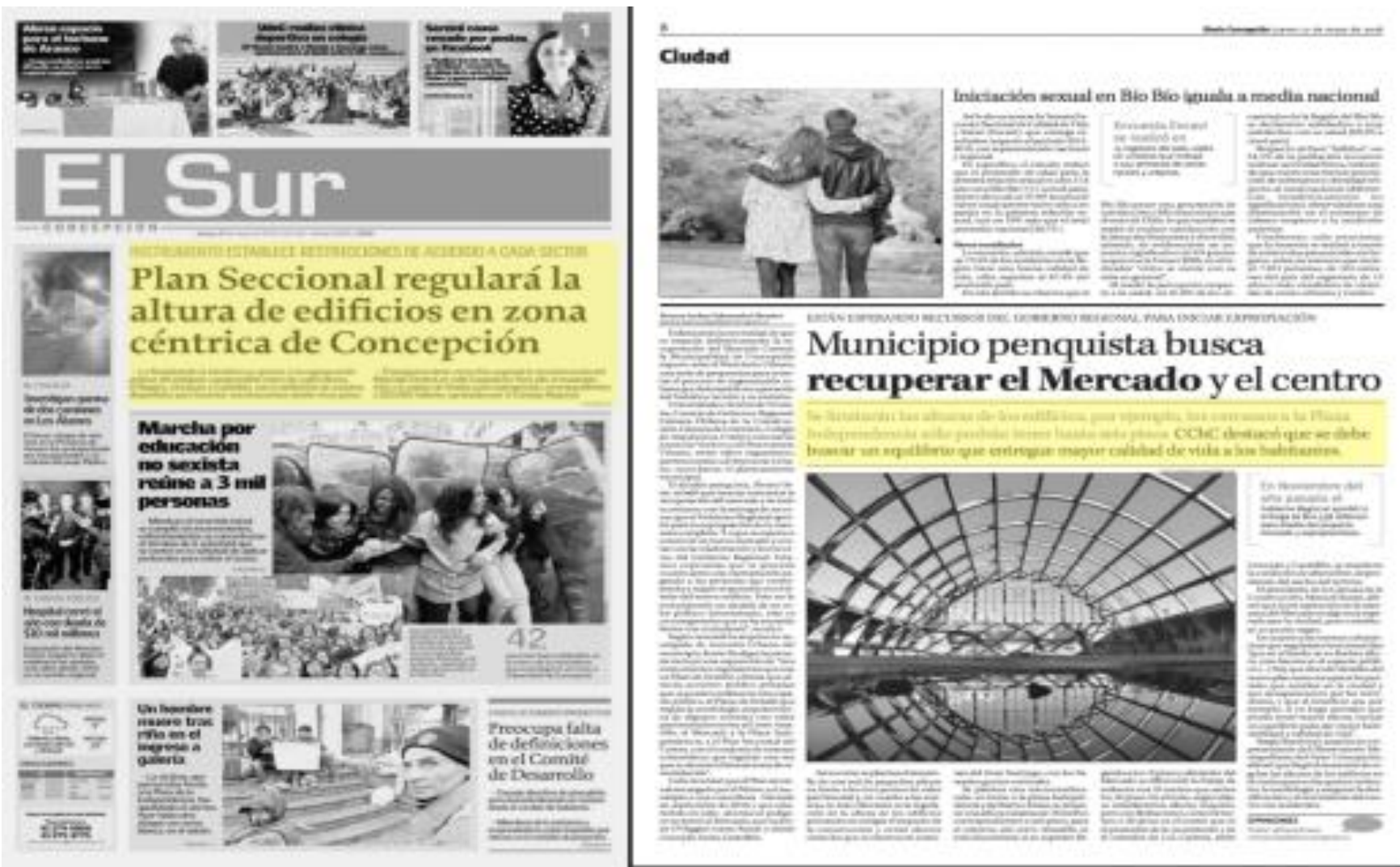

Figura 3. Portada de Diario El Sur y artículo del Diario de Concepción, ambos del 17 de mayo de 2018. Fuente: Elaboración propia.

\section{Metodología}

La investigación se basa en el estudio de caso del Barrio Condell el cual se constituye como pieza urbana representativa por sus valores urbanos, tanto patrimoniales como sociales; que presenta distintos tipos arquitectónicos y se encuentra dentro del polígono de Renovación Urbana. Hasta la fecha, se han construido tres edificios residenciales en altura $y$ un cuarto en construcción, evidenciando procesos de reestructuración espacial en un espacio limitado, similares a los que se desarrollan en otros barrios de Concepción. Todo ello posibilita identificar las externalidades morfológicas que estas construcciones pueden tener en barrios centrales y pericentrales.
La metodología implementada fue cualitativa y cuantitativa de tipo no experimental, con estructura de diseño metodológico de tipo transeccional descriptivo, es decir, se describieron los datos obtenidos en un momento único, excepto al momento de comparar con lo que existía anteriormente dónde sería de tipo longitudinal (Hernández, Fernández y Baptista, 2014).

El análisis cuantitativo considera, en primera instancia, el catastro de los permisos de edificación otorgados entre los años 2010 y 2017 para el polígono de Renovación Urbana, a partir de los cuales se identificaron aquellos otorgados desde 2014, de más de 10 pisos y pertenecientes al polígono de estudio del barrio Condell, para este caso tres construcciones de 16, 17 y 18 pisos, Edificio Janequeo (Janequeo \#874), Edificio Condell (Las 
Heras \#1464) y Edificio Future Live (Ainavillo \#970) respectivamente.

En segunda instancia se construyeron los alzados de fachada de las manzanas más significativas del barrio, incluyendo las que contienen a los edificios previamente seleccionados, para el posterior análisis planimétrico. La selección de estos alzados corresponde a fachadas claves para entender la escala del barrio y las tipologías de viviendas existentes en la actualidad. Posteriormente, se estudiaron comparativamente las manzanas que contienen a los edificios residenciales en altura, determinando la proporción que el edificio ocupa en el terreno y el uso de suelo presente en esa manzana.

Para el análisis cualitativo se clasificaron tres grupos de actores presentes dentro del barrio: a) agentes Inmobiliarios b) agentes residentes nuevos: personas que viven en las edificaciones en altura estudiadas; c) agentes residentes antiguos: residentes y propietarios de las viviendas del Barrio Condell que llevan más de cinco años viviendo en el sector. La muestra fue de tres entrevistas por grupo, a los cuales se les realizó dos preguntas abiertas y con opción del entrevistado de explayarse a voluntad. Las preguntas fueron las siguientes: a) ¿Cómo cree usted que afecta la aparición de torres departamentales en el barrio? ¿Qué opina usted de la diferencia de altura entre las casas existentes y los edificios recientemente construidos? Posteriormente, se procesaron las respuestas utilizando el software de análisis de datos cualitativo ATLAS.ti. y se interpretaron a través del análisis de categorías analíticas, permitiéndonos: (a) asociar códigos o etiquetas con fragmentos de texto (b) buscar códigos de patrones; y (c) clasificarlos (Gallardo Echenique, 2018). Además, desde una perspectiva etnográfica, se recorrió durante el transcurso del año el barrio para reconocer en él elementos que lo hacen característico, principalmente desde su entramado social. En dichos recorridos, asimilados a una deriva urbana (Careri, 2002), se pudo constatar el estado de abandono en que se encuentran distintos lugares y la opinión de vecinos del barrio en cuestión luego de la construcción de estas grandes edificaciones en altura.

\section{Unidad de análisis: Barrio Condell}

La ciudad de Concepción forma parte del plan de Renovación Urbana, siendo el barrio Condell un espacio dentro de la ciudad que se ha caracterizado por tener una relevancia histórica y carácter patrimonial, con una población de 2.662 habitantes según el Censo 2017 (Figura 4). Su eje principal está constituido por la Plaza Condell, que se encuentra entre las calles Janequeo, Bulnes, Ainavillo, Las Heras, Lautaro y avenida Los Carrera. En un sentido más amplio, el barrio Condell comprende el cuadrante Los Carreras, Paicaví, Laguna Las Tres Pascualas y Lientur y, es considerado dentro del sector centro de la ciudad, siendo parte del tejido urbano. Si bien geográficamente el barrio no presenta división con el resto de la ciudad; el espacio social presenta un estado aislado, con cierta distancia del resto de la ciudad. Esta distancia le da cierta interioridad y lo encierra en un espacio o sector que se vuelca a la plaza. Por el lado norte, se encuentra definido en calle Lientur por la escuela Santa Eufrasia (Buen Pastor). Esta institución educacional genera un corte en el sentido de calle Rozas (I. Municipalidad de Concepción, 2011). 

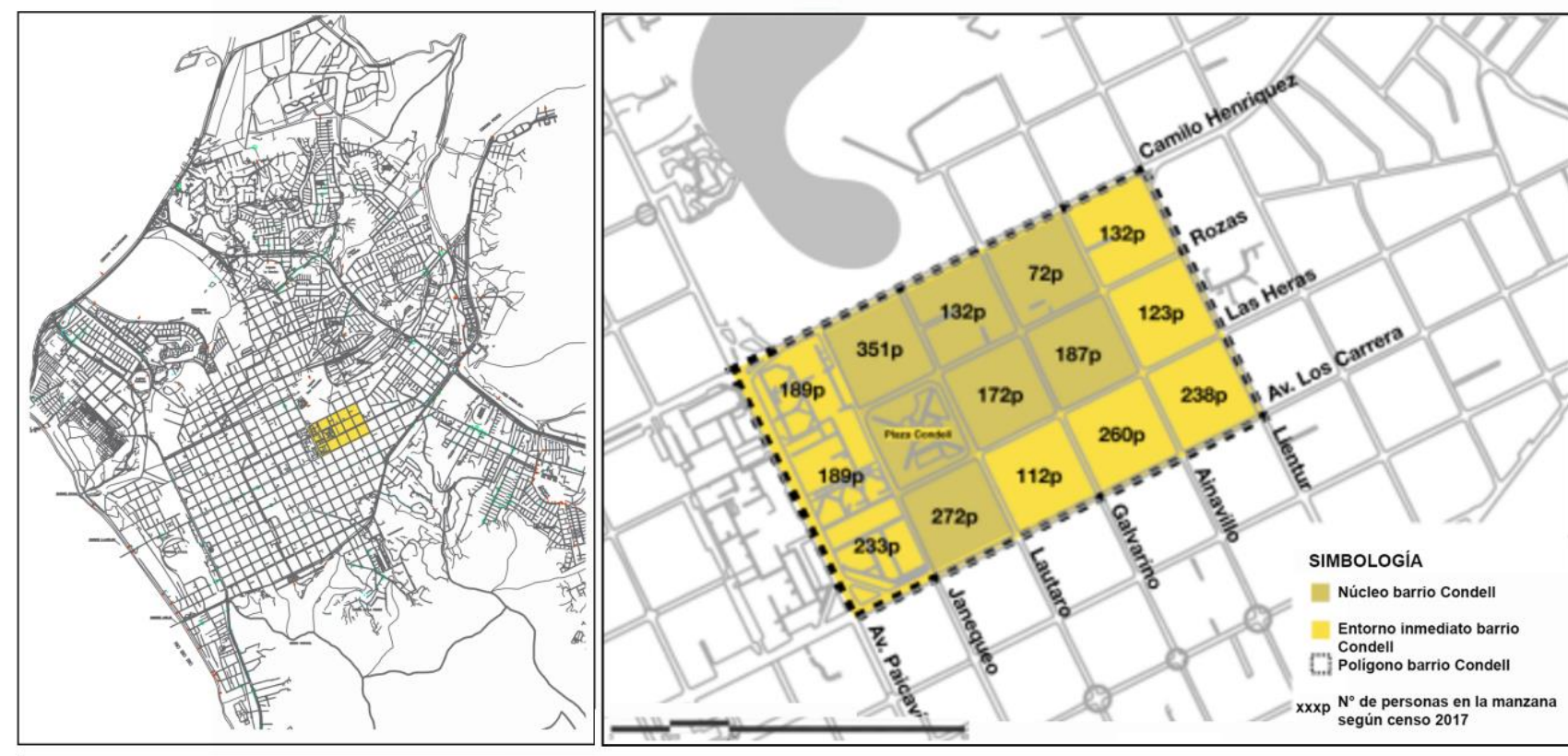

Comuna de Concepción. s/e Ubicación barrio Condell $\square$

Delimitación del barrio Condell por manzana

Figura 4. Delimitación del Barrio Condell en la trama urbana de Concepción en base al polígono definido por la Ilustre Municipalidad de Concepción. № de Personas por manzana del barrio. Fuente: Elaboración propia en base a datos del Censo 2017 entregados por el INE.

La actividad inmobiliaria y la verticalización de la ciudad, en su generalidad trata de edificios en altura de XX en promedio, que se emplazan en sectores de la trama urbana del centro de la ciudad, donde el aprovechamiento al máximo del terreno es el principal objetivo, así como la explotación de múltiples atributos en términos de accesibilidad, cercanía a servicios tales como educación, salud, equipamiento y áreas verdes (Pérez, et al., 2019). Atributos que en el caso del Barrio Condell se encuentran presentes por lo anteriormente mencionado, como es la presencia de vías estructurantes como lo son las calles Paicaví y Los Carrera; áreas verdes como la Plaza Condell, espacios intermedios de la Remodelación Paicaví, la Laguna Las Tres Pascualas; cercanía con centros de salud como Hospital Regional Dr. Guillermo Grant B. y Hospital Clínico del Sur. Por otra parte, en cuanto a equipamientos de educación la presencia de colegios y universidades cercanas también genera atractivo para la población de estudiantes que recibe la ciudad cada año.

\section{Resultados}

Para el caso del edificio Condell de 16 pisos de altura, con 156 unidades habitacionales, el uso de suelo de la manzana representa casi un $18 \%$ de la totalidad, al menos cuatro edificaciones en baja altura anteriores (Figura 5). El edificio Janequeo de 17 pisos de altura, con 150 unidades habitacionales, un ancho hacia la calle Heras de $24 \mathrm{~m}$ y un uso de suelo que representa el $15 \%$ de la totalidad de la superficie total de la manzana (Figura 6). Finalmente, el edificio Future Live, de 18 pisos de altura, con 159 unidades habitacionales y una fachada del ancho total de la manzana, $30 \mathrm{~m}$ ( Figura 7). En general, el entorno de los edificios en estudio, constituyen edificaciones de 2 pisos, reemplazando aproximadamente 4 y 5 edificaciones anteriores de baja altura. 


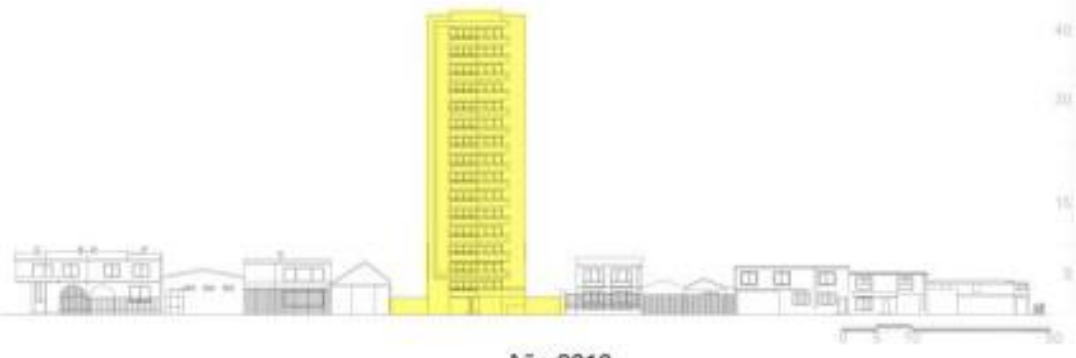

Año 2018

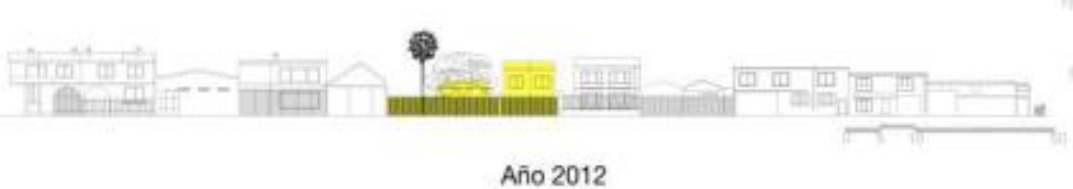

Calle Las Heras, entre calle Lautaro y calle Janequeo.

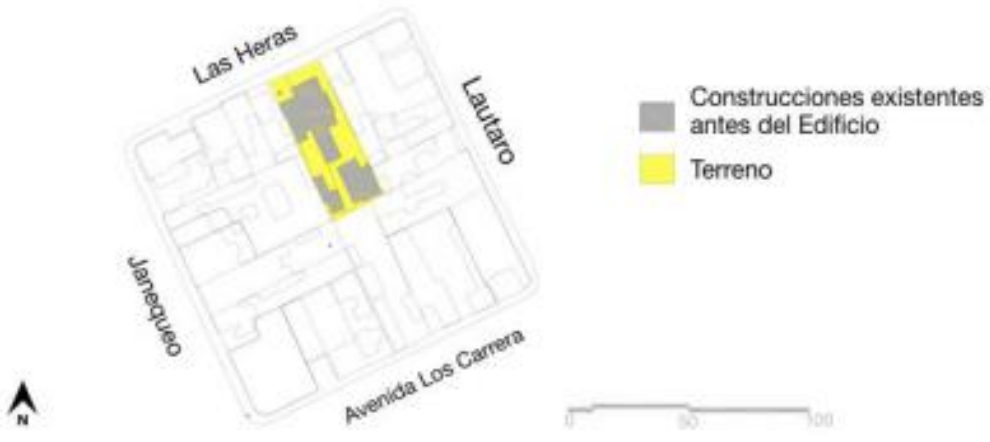

Figura 5. Edificio Condell. Alzada de Fachada: Calle Las Heras, entre calle Lientur y calle Ainavillo. 2012-2018. Fuente: Elaboración propia. 


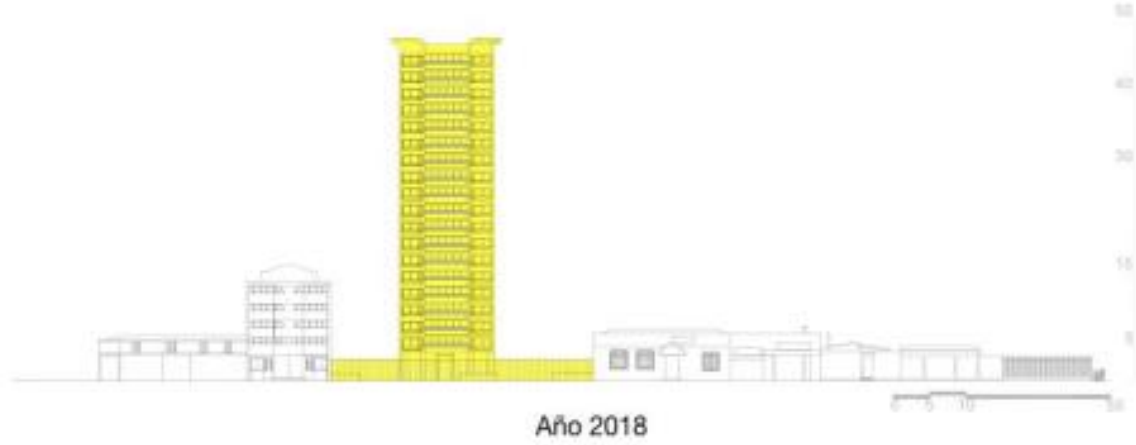

Aగ̂ก 2018

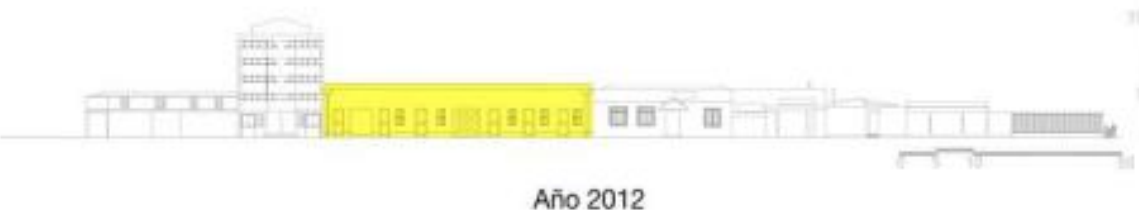

Calle Janequeo entre calle Las Heras y Av. Los Carrera.

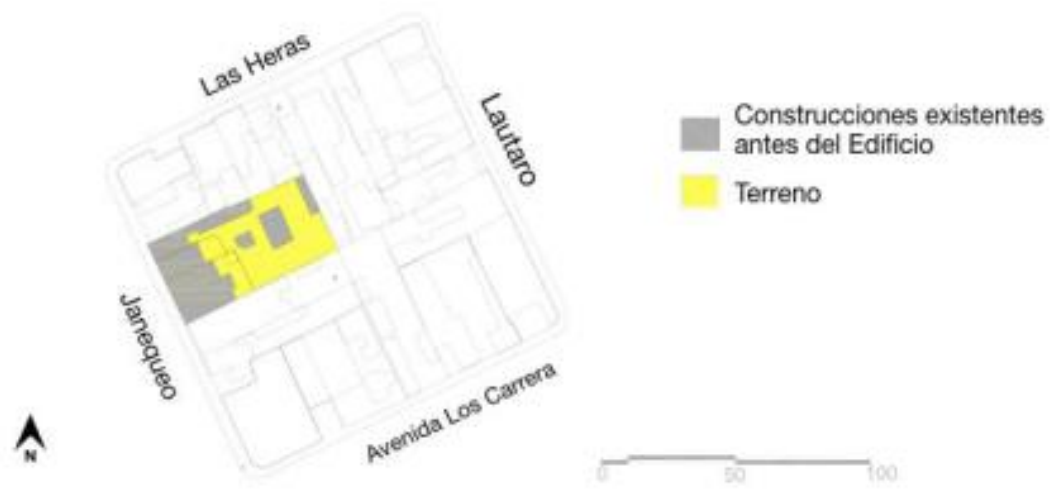

Figura 6. Edificio Janequeo. Alzada de Fachada: Calle Janequeo, entre calle Las Heras y Av. Los Carrera. 2012-2018. Fuente: Elaboración propia. 


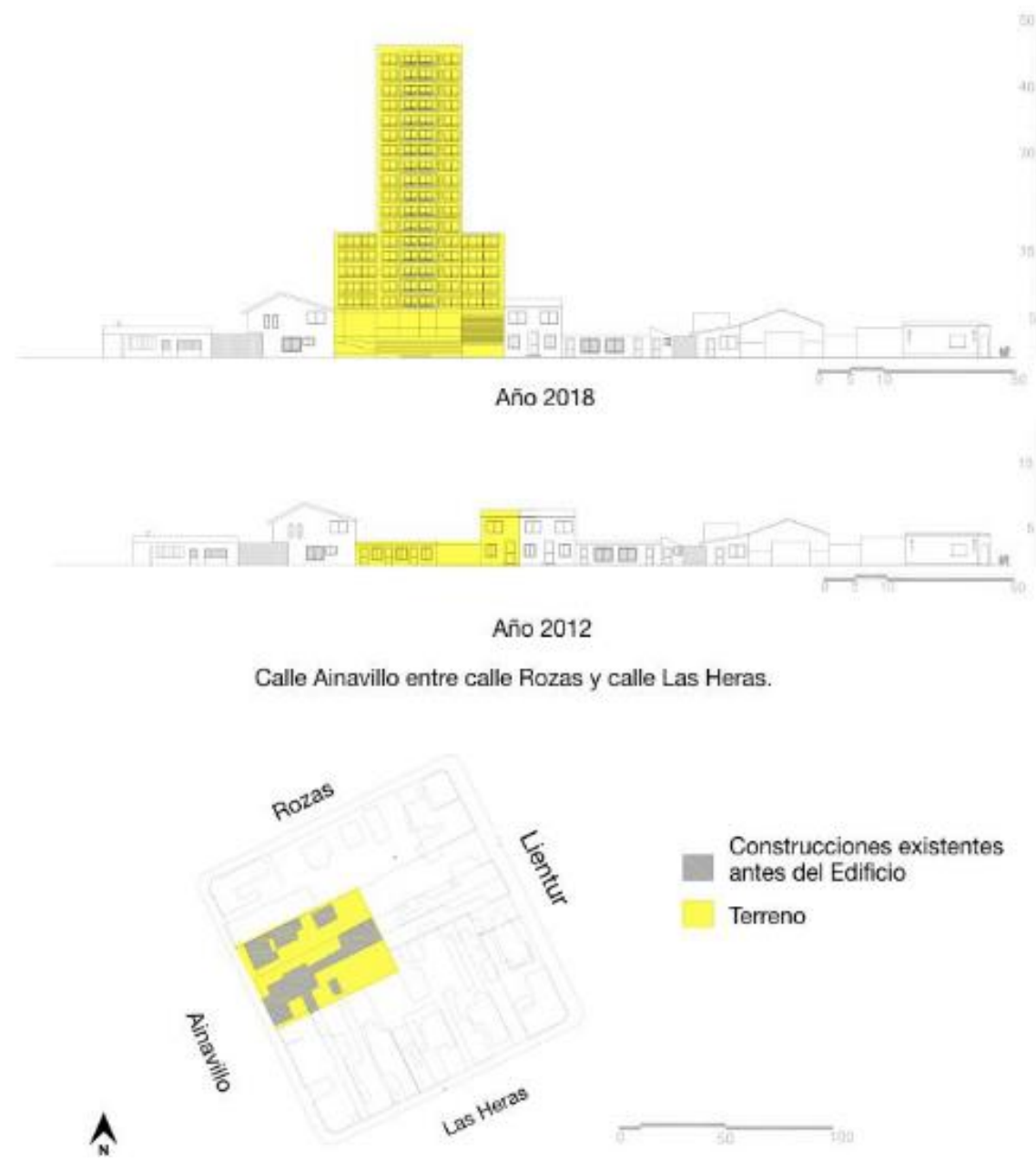

Figura 7. Edificio Future Live. Alzada de Fachada: Calle Ainavillo, entre calle Las Heras y calle Rozas. 2012-2018. Fuente: Elaboración propia.

La presencia de viviendas de 1 y 2 pisos hace que el barrio tenga una escala de no más de $12 \mathrm{~m}$, ya que el promedio de las edificaciones es de entre 9 y $10 \mathrm{~m}$ de altura.

El análisis de los alzados de fachada permite visualizar la implantación de los "edificios residenciales en altura" (de ahora en adelante ERA) dentro de un contexto arquitectónico totalmente distinto a la tipología departamental; además de posicionarse de forma aleatoria en predios fusionados donde antes existían edificaciones acordes a la escala barrial (Pérez, et al., 2019). Lo anterior concuerda con la definición que hace Fedele para los ERA: "unidades desagregadas, dividas y dispersas en el tejido urbano" (Fedele y Martínez, 2015, p.
76). La aparición de los ERA, en al menos tres manzanas, dentro del barrio Condell (con proyección a una cuarta manzana debido al inicio de la construcción de un nuevo proyecto inmobiliario residencial en altura), produce efectos a nivel morfológicos variados.

El análisis cualitativo se realizó mediante entrevistas a residentes antiguos del barrio junto con personas que viven dentro de los nuevos edificios. Las relaciones entre las entrevistas efectuadas y su contenido se resumen en las siguientes redes de códigos, que permiten obtener deducciones y la percepción general de los habitantes frente a las transformaciones que enfrenta el barrio (Figura 8). 


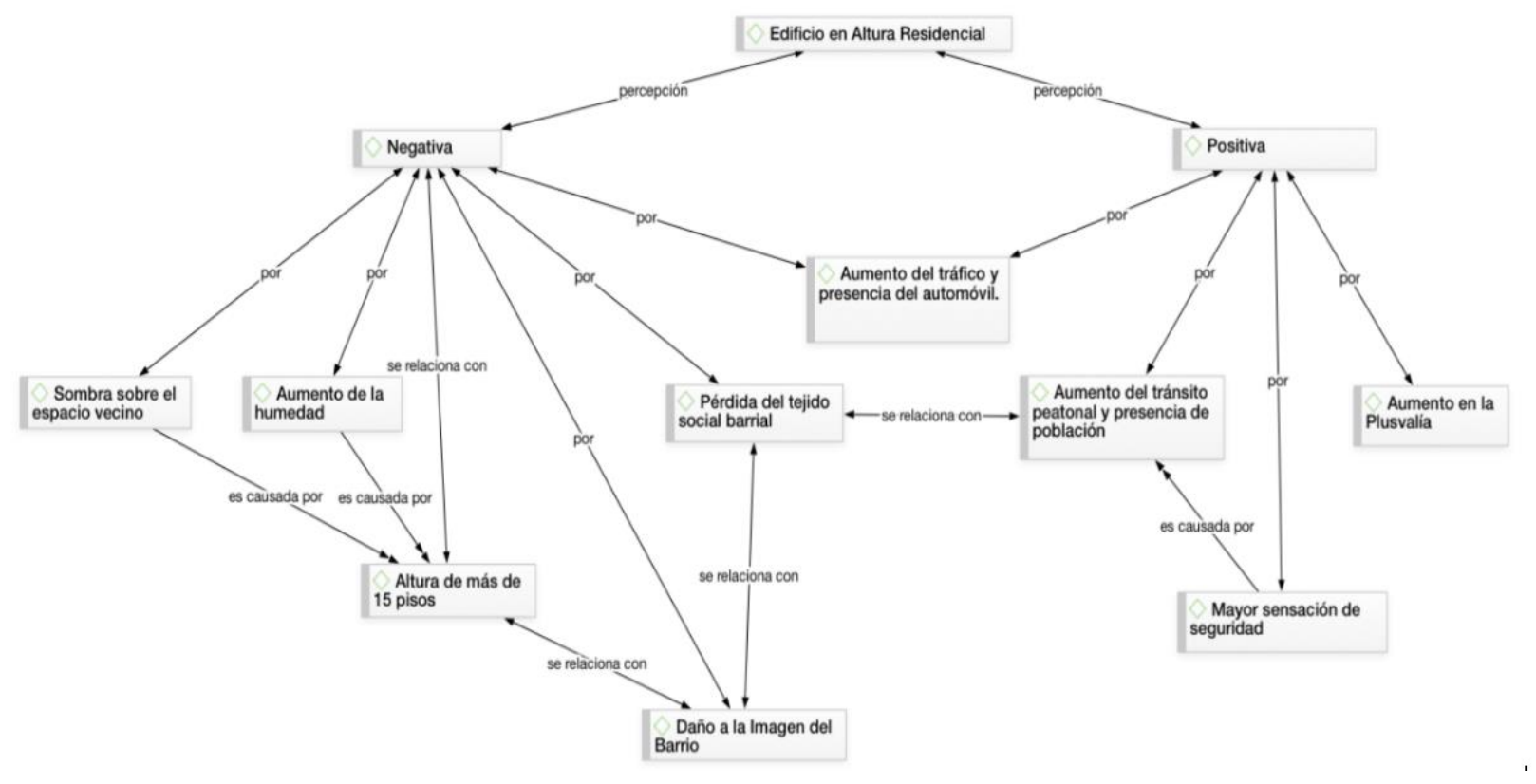

Figura 8. Red de códigos a partir de Atlas.ti. Fuente: Elaboración propia.

El diferencial de altura entre las edificaciones existentes y nuevas es mayor a $35 \mathrm{~m}$, lo que tiene un efecto en la escala del barrio, alterando el patrón de mediana y baja altura que este barrio consolidado posee (Pérez et al., 2019). Los resultados evidencian esto en la calificación negativa para el barrio, por parte de residentes antiguos y nuevos, los que son capaces de reconocer que produce efectos físicos, destacando la alteración en el asoleamiento y la ventilación de las viviendas y espacios públicos que están aledaños, estos últimos pierden luz y permaneciendo algunas en constante sombra, como lo expresa un residente nuevo

\section{[...] personalmente creo que impactan} visualmente, tapan el paisaje, hacen sombra y también hace que pierda la estructura o la estética que tenía el barrio. Porque son edificios distintos a lo que sucede en la remodelación Paicaví o a las casas alrededor de la plaza.

El $100 \%$ de la muestra manifiesta en forma negativa la altura de los edificios, y son categóricos al aspecto. Un residente que ha vivido por 56 años manifiesta: "Opino que nos sentimos aplastados, nos quitan el sol. Siento que nos quitan oxígeno y vida". Estos efectos se sienten de mayor forma en las edificaciones contiguas a los ERA, dado que proyectan un cono de sombra mayor, legalmente aceptada por el cálculo del cono de sombra, que termina por sumergir en humedad a los vecinos: "la humedad me afecta porque no me pega el sol en toda la tarde. Entonces el frío es fuerte, uno que no estaba acostumbrado, que tenía solcito para secar la ropa en el patio. Claro que afecta".

Los tres proyectos inmobiliarios ocupan alrededor del $15 \%$ de la manzana que los contiene, no obstante, constituyen los sitios donde más población vive dentro del barrio. Cada uno de los edificios se posiciona en un predio que se formó a partir de la fusión de otros más pequeños, que contenían una edificación residencial de poca altura y baja densidad. Esto reafirma el proceso de verticalización que está viviendo el Barrio Condell, donde se reemplazan edificaciones a raíz de una creciente actividad inmobiliaria en altura, afectando las formas y estructuras tradicionales e históricas, además de afectar la vida de los barrios (Pérez, et al., 2019).

Las entrevistas a actores clave dan cuenta de las variables a estudiar en profundidad ampliando la mirada de esta investigación a partir de encuestas otorgando representatividad muestral

Las opiniones, frente a la presencia de ERA dentro del barrio, constituyen percepciones negativas, independiente si el entrevistado es un vecino del barrio o alguien que se habitante de los edificios. Esto coincide con lo que Fedele expresaba frente al ERA: 
Es así que el indicador simbólico de proceso de la forma urbana deja de condensarse en la única presencia del edificio en altura, para desplazarse a otros aspectos que justamente lo rechazan: el respeto por el patrimonio, la preservación de características morfológicas del tejido existente y los usos del espacio urbano. (Fedele y Martínez, 2015)

Estas percepciones negativas provienen en su mayoría de problemas generados por la diferencia de altura entre las edificaciones y por el aumento de la población y el tráfico vehicular. No obstante, es interesante conocer cómo las opiniones entre ambos grupos difieren en ciertos puntos. En el caso de la seguridad, tema abordado por los propios entrevistados, al menos el $50 \%$ de los vecinos del barrio sienten que se pierde la sensación de confianza y seguridad por la llegada en masa de nuevas personas al barrio, esto en contraste con la opinión en su totalidad de los habitantes de los edificios, quienes plantean que el mayor flujo de gente asegura mayor seguridad al espacio público y por ende al sector.

De las treinta personas entrevistadas, al menos veinte consideraron al barrio como un lugar muy privilegiado respecto a su cercanía con el centro, pero a la vez la tranquilidad que rodea el lugar, a pesar del aumento del tráfico y la lucha constante por estacionamiento. Además, la presencia de la plaza como espacio recreativo y área verde influye mucho en el por qué eligieron el barrio Condell para vivir. Señalan, además, que estos atributos son los que también hacen que las inmobiliarias vean en el barrio un buen lugar para invertir; al menos cuatro vecinos del barrio declararon que las empresas les han ofrecido dinero para la venta de su vivienda.

Finalmente, se han logrado constatar singularidades en la percepción de los distintos grupos analizados respecto a cómo se construye hábitat. Así, antiguos residentes consideran que viviendas no aledañas a los edificios se ven valorizadas económicamente, en contraposición, viviendas aledañas se desvalorizan a causa de factores morfológicos que impactan negativamente en la calidad de vida.

Por otro lado, la percepción de los habitantes de los ERA difiere en mayor medida de antiguos residentes respecto a edificaciones en altura en el barrio, estos no visibilizan perdida de unidad barrial, sin embargo, reconocen al edificio en altura como elemento de mayor escala a la existente, que irrumpe en la morfología del barrio, no obstante, priorizan la necesidad de cercanía al centro urbano y los beneficios de la proximidad.

En este sentido, a nivel del habitar cotidiano, habitantes de los ERA utilizan la plaza del barrio en menor medida que los antiguos residentes, manifestando cierta preferencia por espacios que están situados cerca del barrio, con patrones de desplazamiento cotidiano vinculados a redes sociales existentes fuera del barrio, de mayor alcance territorial. aquí, sus preferencias de ocio y consumo están fuera del barrio, pero dentro de la comuna de Concepción, como el Parque Ecuador, el centro histórico y lugares que valorizan las vías estructurantes que permiten acceso principalmente por la caminata. Sin embargo, se observa que lo anterior no ha decantado en la perdida de vida de barrio, como declara un residente nuevo: "hay cambios respecto al movimiento de gente, al comercio, aun así, creo que sigue habiendo vida de barrio".

\section{Conclusiones}

El análisis del caso viene a confirmar que el Barrio Condell está siendo afectado por un proceso de verticalización, expresado en la densificación de sus manzanas y la aparición de proyectos inmobiliarios en altura, caracterizando el proceso como un reemplazo de viviendas existentes y fusión predial, la construcción de un edificio residencial en altura de más de 10 pisos, y el aumento de la población y el tráfico.

El proceso de verticalización genera, a nivel morfológico urbano, transformaciones en la escala del barrio quebrando la actual escala compacta y de baja y media altura, donde la mayoría de sus edificaciones residenciales son de uno o dos pisos, con la presencia de edificios de 5 a 8 pisos. La instalación de estas tres torres de departamentos de 16, 17 y 18 pisos en el barrio se oponen al tejido y a las tipologías arquitectónicas características del barrio, lo que produce un quiebre de la forma urbana y el espacio público.

La percepción de los habitantes antiguos del Barrio es en general negativa frente a la inserción de estos edificios en el tejido urbano del barrio Condell. Los entrevistados identifican en la altura de los edificios la raíz de los principales problemas físicos que traen estos proyectos 
inmobiliarios. Los aproximadamente más de $40 \mathrm{~m}$ de edificio producen una sombra que se proyecta sobre las edificaciones vecinas y el espacio público, sumado a una diferencia de altura considerable entre las edificaciones hace que la humedad comience a aparecer en los espacios donde la luz y el calor del sol no llegan. Incluso en el otro sentido, quienes viven en las torres identifican problemas con la diferencia de altura entre edificaciones, es el caso de quienes viven en departamentos cercanos al techo de la vivienda vecina quien por ejemplo evidencian problemas con el humo que producen las estufas de las casas vecinas, generando problemas al momento de abrir la ventana.

Los vecinos del barrio están conscientes que el Barrio Condell posee una historia y un carácter propio que consideran no será fácil de destruir, y que finalmente constituye uno de los atractivos propios del lugar. Existe preocupación de parte de los residentes antiguos del barrio frente a la llegada masiva de personas considerando difícil generar lazos de vecindad como los que existen dentro del sector.

Resulta llamativo que quienes viven dentro de las torres de departamentos, también identifican negativamente la inserción de estos proyectos dentro del barrio. Son conscientes en su mayoría de la diferencia de altura e imagen que presentan estos edificios frente a lo que ya existía en el barrio. Quienes llegan a vivir al Barrio Condell, creen que la tranquilidad y la cercanía con el centro constituye atractivos fundamentales para residir en este barrio. El aumento de la población y el tráfico a través de este proceso de densificación genera opiniones divididas al respecto, dónde existe quienes tienen la percepción que el aumento de la población también trae consigo mayor seguridad y vigilancia policial al barrio. Abriendo una arista interesante de análisis frente a las percepciones de seguridad dentro del barrio y que hasta el momento no se estaba considerando.

De estas forma, la presentes investigación exploratoria sobre los procesos de verticalización en el centro urbano de la ciudad de Concepción abre nuevas aristas para ampliar respecto a variables que fueron relevadas. Dentro de ellas, resulta de interés entender como este proceso de reestructuración urbana central afecta las prácticas de movilidad del barrio, además de conocer cómo y en qué medios se desplazan a distintas actividades urbanas tanto residentes nuevos como antiguos, evaluar su nivel de acceso y apropiación de movilidad en contexto de reestructuración urbana [B]

\section{Bibliografía}

Allen, E. (2013). Cómo funciona un edificio. Principios elementales. Barcelona: Gustavo Gili.

Brenner, N. (2004). New States Spaces. Urban Gobernance and the Rescaling of Statehood. New York: Oxford

Brenner, N. y Theodore, N. (2002) Cities and the geographies of "actually existing neoliberalism". Antipode. 34(3), 349-379. http://dx.doi.org/10.1111/1467-8330.00246.

Careri, F. (2002). Walkscapes. El andar como práctica estética. Barcelona: Gustavo Gili.

Cisterna J. (05 de mayo 2018). El Gran Concepción y sus cambios en los últimos 15 años. Diario de Concepción.

Clark, E. (2005). The order and simplicity of gentrification: A political challenge. In Loretta Lees, Tom Slater \&
Elvin Wyly, The Gentrification Reader, pp.24-31. London.

Colegio de Arquitectos de Chile. Iquique (2018). Causas y efectos urbanos de la verticalización inmobiliaria. Recuperada de http://colegioarquitectos.com/noticias/?p=20596

Costa, A. (2000). A verticalização de natal: elemento de transfor-mações sócio-espaciais. Ponencia presentada en VI Seminário de História da Cidade e do Urbanismo. Universidade Federal do Rio Grande do Norte, Brasil

Cuthbert, A. (2006). The Form of Cities. Political Economy and Urban Design. Oxford: Blackwell.

De Mattos, C. (2004). Globalización y urbanización en América Latina. 
Fedele, J. y Martínez, I. (2015). Verticalización y desarrollo inmobiliario del núcleo urbano central de Santa Fe: Cambios morfológicos, conflictos urbanos y regulaciones edilicias en la recuperación postcrisis 2001. Cuaderno Urbano. Espacio, Cultura, Sociedad, 18(18), 65-88. Disponible en https://revistas.unne.edu.ar/index.php/crn/article/vi ew/265

Gallardo Echenique, E. (2018). Utilización del programa de análisis cualitativo ATLAS.ti para gestionar y analizar datos. Recuperado de https://atlasti.com/2014/06/12/utilizacion-delprograma-de-analisis-cualitativo-atlas-ti-paragestionar-y-analizar-datos/

Gehl, J. (2010). Cities for people. London: Island Press.

Gehl, J. (2011). Life between buildings. Using public space. London: Island Press.

Harvey, D. (1989). From managerialism to entrepreneurialism: the transformation in urban governance in late capitalism. Geografiska Annaler: Series B, Human Geography, 71(1), 3-17. http://doi.org/10.1080/04353684.1989.11879583

Hernández, R., Fernández, C., y Baptista, M. (2014). Metodología de la investigación. México: McGrawHill.

Hidalgo, R. y Janoschka, M. (2014) (Eds). La Ciudad Neoliberal. Gentrificación y exclusión en Santiago de Chile, Buenos Aires, Ciudad de México y Madrid. Santiago de Chile/Madrid: Serie GEO-libros, Instituto de Geografía, Pontificia Universidad Católica de Chile; Departamento de Ciencia Política y Relaciones Internacionales, Universidad Autónoma de Madrid

Ilustre Municipalidad de Concepción. (2011) Programa Quiero Mi Barrio: Barrio Condell.

http://quieromibarrio.cl/index.php/biobio/

Ingold, T. (2007). Lines: A brief history. London: Routledge.

Ingold, T. (2013). Making: Anthropology, archaeology, art and architecture. London: Routledge.

Instituto Nacional de Estadísticas (2017). Censo 2017. Síntesis de resultados del Censo. Santiago de Chile: INE.

Instituto Nacional de Estadísticas (2018). Galería de Mapas. Recuperado de http://www.ine.cl/herramientas/galeria-demapas/inicio
Inzulza, J. y Galleguillos, X. (2014). Latino gentrificación y polarización: transformaciones socioespaciales en barrios pericentrales y periféricos de Santiago, Chile. Revista Geografía Norte Grande, 58, 135-159. https://doi.org/10.4067/s0718-34022014000200008

Janoschka, M. (2011). Geografías urbanas en la era del neoliberalismo. Una conceptualización de la resistencia local a través de la participación y la ciudadanía urbana. Investigaciones geográficas, (76), 118-132.

Klein, N. y García, I. F. (2007). La doctrina del shock: el auge del capitalismo del desastre. Barcelona: Paidós.

Lefebvre, H. (1968). Le droit à la ville. Paris: Anthropos

López-Morales, E. (2013). Gentrificación en Chile: aportes conceptuales y evidencias para una discusión necesaria. Revista de Geografía Norte Grande, (56), 31-52. https://doi.org/10.4067/s0718$\underline{34022013000300003}$

López-Morales, E., Gasic, I., y Meza, D. (2012). Urbanismo pro-empresarial en Chile: políticas y planificación de la producción residencial en altura en el pericentro del Gran Santiago. Revista INVI, 27(76), 75-114. https://doi.org/10.4067/s0718-83582012000300003

López-Morales, E., Gasic, I., y Meza, D. (2014). Neoliberalismo, regulación ad-hoc de suelo y gentrificación: el historial de la renovación urbana del sector Santa Isabel, Santiago. Revista de geografía Norte Grande (58), 161-177.

Ortiz, J., Castro, C. y Escolano, S. (2002). Procesos de reestructuración urbana y niveles de vulnerabilidad a amenazas naturales en una ciudad de tamaño medio: La Serena, Chile. Investigaciones Geográficas (36), 1741. https://doi:10.5354/0719-5370.2013.27744

Pérez, L. (20 de agosto de2017). Renovación urbana y Desplazamiento. Diario El Sur, p. 3. Recuperado de http://www.elsur.cl/impresa/2017/08/20/papel/

Perez, L., Hidalgo, R., Añasco, L., y Alvarado, V. (2019). Renovación urbana, dinámicas inmobiliarias y cambio socioeconómico en las áreas centrales del Gran Concepción, Chile. El sector Ribera Norte. Manuscrito no publicado.

Sabatini, F. (2000). Reforma de los mercados de suelo en Santiago, Chile: efectos sobre los precios de la tierra y la segregación residencial. Eure (Santiago), 26(77), 49-80. https://doi.org/10.4067/s025071612000007700003 
Sequera, J., \& Janoschka, M. (2015). Gentrification dispositifs in the historic centre of Madrid: A reconsideration of urban governmentality and state-led urban reconfiguration. Global gentrifications: Uneven development and displacement, 375-394.

Silva, L. (2004). São Paulo de 1930 a 1945: verticalização, expansãohorizontal e grandes obras viárias. Ponencia presentada en VIII Seminário de Históriada Cidade e do Urbanismo, Rio de Janeiro, Brasil.
Vergara Vidal, J. (2017). Verticalización. La edificación en altura en la Región Metropolitana de Santiago. INVI, 32(90), 9-49. https://doi.org/10.4067/s071883582017000200009 